\title{
Mechanical stirring influence on solute segregation during plane front directional solidification
}

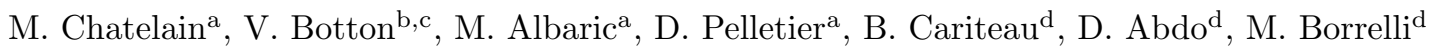 \\ ${ }^{a}$ Univ. Grenoble Alpes, INES, F-73375 Le Bourget du Lac, France \\ CEA, LITEN, Department of Solar Technologies, F-73375 Le Bourget du Lac, France \\ ${ }^{b}$ Laboratoire de Mécanique des Fluides et d'Acoustique, CNRS/Université de Lyon, Ecole Centrale de Lyon/Université Lyon 1/INSA \\ Lyon, ECL, 36 Avenue Guy de Collongue, 69134 Ecully Cedex, France \\ ${ }^{c}$ INSA Euromed, Université Euro-Méditerranéenne de Fès, route de Meknes BP 51, 30000 Fez, Morocco. \\ ${ }^{d}$ DEN/DANS/DM2S/STMF/LIEFT, CEA Saclay 91191 Gif-sur-Yvette Cedex
}

\begin{abstract}
The present paper focuses on directional solidification processes for photovoltaic silicon purification. The use of a mechanical stirrer in the melt to enhance impurity segregation is investigated through numerical simulations. The 3D forced convection flow is resolved in a transient regime thanks to a sliding mesh approach. The hydrodynamic model is coupled to a solute transport simulation in a quasi-steady approximation (i.e. with constant liquid height). Velocity measurements are performed by Particle Image Velocimetry on a water model in order to validate hydrodynamic simulations. Numerical results show that an efficient segregation can be achieved, even for high solidification rates, thanks to mechanical stirring. The numerical model provides meaningful insights for process optimization as it correlates the impurity repartition on the solidification front to the stirring parameters. Finally, the numerical segregation results are compared to an analytical model of the solute boundary layer. It is found that the analytical model provides a good estimate of the mean segregation regime from an hydrodynamic simulation of the forced convection flow, which makes it a useful tool for process design.
\end{abstract}

Keywords: directional solidification, segregation, mechanical stirring, convection, silicon purification

\section{Nomenclature}

$\Delta$

$\delta$

$\mu$

$\mu_{t}$

$\nu$

$\nu_{t}$

$\rho$

$\tau$

$\vec{u}$

$B$

$c$

$C_{L}$

$C_{S}$

$D$ convecto-diffusive parameter

solute boundary layer thickness

dynamic viscosity

turbulent dynamic viscosity

.

kinematic viscosity

turbulent kinematic viscosity

density

wall shear-stress

fluid velocity

dimensionless segregation parameter

distance from impeller to solidification front . (m)

solute mass fraction in the liquid

solute mass fraction in the solid

molecular diffusivity
(-) $d$

. (m) $D_{t}$

(Pa.s)

(Pa.s) .. $\left(\mathrm{m}^{2} / \mathrm{s}\right)$ . $\left(\mathrm{m}^{2} / \mathrm{s}\right)$ $\ldots\left(\mathrm{kg} / \mathrm{m}^{3}\right)$

$p$

$R e_{d}$ Reynolds number

Sc Schmidt number

$S c_{t}$

$V_{I}$

impeller diameter

$L \quad$ crucible width

$N \quad$ rotational speed

pressure

interface velocity turbulent diffusivity $\left(\mathrm{m}^{2} / \mathrm{s}\right)$

Fq dimensionless pulsation

$H \quad$ liquid silicon height

$k_{0} \quad$ segregation coefficient

$k_{\text {eff }}$ effective segregation coefficient $\ldots \ldots \ldots \ldots \ldots$. (-) $(\mathrm{rpm})$

turbulent Schmidt number 


\section{Introduction}

\subsection{Context}

The silicon purification is an important issue for the photovoltaic industry since impurity concentrations have a strong influence on solar cells efficiency [1, 2]. In order to reduce costs and environmental impact, specific purification processes, based on metallurgical operations, are developed for photovoltaic silicon [3]. The segregation phenomenon, which occurs during a directional solidification process, is an efficient way to remove metallic impurities which are usually far less soluble in solid silicon than in liquid silicon and thus feature low partition coefficients [4]. An intense convection in the melt is, however, required to ensure efficient segregation, especially if high growth rates are targeted, for productivity sake. Various stirring techniques can then be used during directional solidification processes to control fluid flow and improve material quality.

Electromagnetic stirring is already widely used for Czochralski processes, in order to tailor convection and control dopants and oxygen incorporation [5-7]. This technique is also investigated for directional solidification processes [8-11]. Nevertheless, skin effect is expected to limit the velocity magnitude in the bulk of the melt for large ingots [12]. Mechanical stirring is an interesting solution allowing high velocities and strong turbulence in the melt, which is desirable for an efficient segregation in large industrial ingots (crucible width in the order of 1 meter). This technique is widely used in chemical industries for mixing operations [13, 14] and for some metallurgical processes like aluminum degassing [15-17]. This solution was recently investigated for silicon directional solidification [12, 18]. Other stirring techniques can also be cited, such as acoustic stirring [19-21] or the use of steady magnetic fields combined with electrical current injection [22]. The present paper focuses on a pilot scale directional solidification process (crucible width $38 \mathrm{~cm}$ ) using an axial impeller to ensure an efficient segregation of metallic impurities.

\subsection{State of the art}

The simulation of fluid convection flow and solute transport in the melt represents an efficient optimization tool for the silicon purification process by segregation. In the literature, the simulation of rotational impellers has been widely investigated, especially for chemical processes applications. Different methods have been proposed in order to predict fluid motion and turbulence properties in stirred tanks. Readers are referred to Aubin et al. [13] and Brucato et al. [14] for a review of the different methods. At first, empirical models, like Impeller Boundary Conditions (IBC) or momentum source terms models, were proposed. Such simplified approaches rely on experimental data and provide no information on the flow in the impeller region. Then, with the increase of computational capacities, more sophisticated methods, describing the exact geometry of the impeller, were proposed. The Multiple Frames of Reference (MFR) approach was first introduced by Luo et al. [23]. In this approach, the computational domain is divided in two zones by a cylindrical interface encompassing the impeller. In the impeller region the flow is computed in a frame rotating with the impeller, and in the outer region it is computed in a fixed reference frame. Aubin et al. [13] explain that this method is a steady-state approximation which is valid when the distance between the tank walls and the impeller blades is sufficiently large to assume that the tank geometry has no influence on the flow in the impeller region. For a complete transient resolution of the flow, a sliding mesh technique must be used to simulate the impeller rotation. This approach requires more computational means, but involves no assumption on the impeller flow and its interaction with the tank. In these problems, turbulence description often represents a major issue. As a matter of fact, turbulence has a direct influence on mixing processes. Recent studies focus on this aspect and investigate the potential of advanced turbulence models, like Large Eddy Simulation (LES), to enhance stirring models performances [24, 25]. Once again, these developments are made possible by the increase of computational capacities.

Experimental characterization of stirred tanks is also widely investigated in the literature [26]. The aim is to provide physical understanding of the transfer processes and to improve and validate numerical models. Regarding velocity field measurements, optical techniques are usually preferred. For instance, Laser Doppler Velocimetry (LDV) is often used to produce precise measurements near the impeller because it provides a high spatial and temporal resolution [26]. Alternatively, Particle Image Velocimetry (PIV) is an efficient technique which gives access to the velocity field on a complete plane of the flow. This aspect makes it a very suitable technique for the characterisation of global flow structure in stirred tanks [27-29]. Metallurgical processes can also be investigated thanks to experimental models, providing that right similarity conditions are defined. As an example, CamachoMartínez et al. [15] investigated a pilot system of aluminum degassing using a full scale water physical model. PIV measurements were performed to determine flow patterns and turbulence characteristics in the stirred tank.

On the other hand, several numerical studies are dedicated to the impact of convection flow on directional solidification and solute segregation [8, 9, 30-32], but few involve a mechanical stirrer. Dumitrica et al. [12] performed 
a numerical investigation of mechanical stirring influence on a silicon directional solidification process. Authors used momentum source terms to reproduce the action of a cylindrical stirrer (tangential velocity component). The aim of the study was to highlight the influence of the rotation speed on the solid/liquid interface deflection. Analytical approaches are also developed in order to estimate the solute boundary layer from the knowledge of the melt convection flow. Recently, analytical models of the solute boundary layer have been proposed by Altenberend et al. [33] in the case of 1D unbounded turbulent parallel flows and Garandet et al. [34] for a 2D laterally confined flow. This last model is derived from a scaling analysis procedure previously introduced for the study of segregation under natural convection in Czochralski and horizontal Bridgman growth configurations [35]. The aim of this analytical model is to provide an estimate of the segregation regime, without performing a complete simulation of the segregation problem. The finality is to avoid a numerical resolution of the solute boundary layer, which can be demanding in computational means. An extension of this model for fully turbulent configurations, with significant effects of the turbulent transport inside the solute boundary layer, has been proposed in a recent publication [36]. Up to now, this model has been tested in a canonical reference case, namely the 2D lid driven cavity, both in quasi-steady and transient regimes. Kaddeche et al. [37] also compared the analytical model to numerical and experimental results of segregation under natural convection in horizontal Bridgman configurations.

\subsection{Objective of the study}

The aim of this work is to propose numerical and analytical tools for the study of solute segregation under mechanical stirring. The present study focuses on the application of an axial impeller for a silicon purification process. In this frame of work, fluid flow must be precisely described in order to get a realistic picture of the solute transport in the liquid phase. 3D transient simulations of the flow generated by the axial impeller are, therefore, performed using a sliding mesh approach. An experimental characterization of the velocity field is performed by PIV measurements on a water experiment model for the validation of the stirring simulations. The hydrodynamic simulations are coupled to a solute transport computation. Segregation is an inherently transient phenomenon since solute rejection into the liquid phase is generated by the displacement of the solidification front. The representative time scale of the solidification process is, however, much larger than the time scale associated with the rotation of the impeller. A quasi-steady assumption is thus adopted regarding the solidification process, as formerly proposed in several studies [34,38-42]. This numerical model is used to investigate the influence of stirring parameters on the solute boundary layer at the growth front. Finally, numerical segregation results are compared to an analytical model of the solute boundary layer. The purpose of this approach is to allow the estimation of the segregation regime from a purely hydrodynamic simulation.

\section{Numerical procedure}

\subsection{Present configuration and segregation concepts}

We consider a silicon directional solidification process. The present study is dedicated to a pilot scale purification process of photovoltaic silicon by segregation during directional solidification. A schematic representation of the investigated configuration is presented in figure 1. We consider a square shaped crucible, of width $L=38 \mathrm{~cm}$, filled with silicon. The initial liquid silicon height in the crucible, denoted $H$, is about $20 \mathrm{~cm}$ for a typical load of $70 \mathrm{~kg}$. The reference frame is attached to the solidification front, $z$ being the normal to the solid/liquid interface pointing toward the liquid. The solidification front is assumed to be plane and its velocity is denoted $V_{I}(\mathrm{~m} / \mathrm{s})$. An axial impeller, with non standard geometry, is immersed in the melt. The impeller comprises 3 blades, its diameter is $d=13 \mathrm{~cm}$ and its rotational speed is denoted $N(\mathrm{rpm})$. During the solidification process, the distance between the impeller and the solidification front, denoted $c$, is kept, as far as possible, constant and equal to $5 \mathrm{~cm}$. This distance is difficult to control precisely in directional solidification experiments and the arbitrary value of $c=5 \mathrm{~cm}$ reflects the necessity of a safety margin regarding this parameter. 


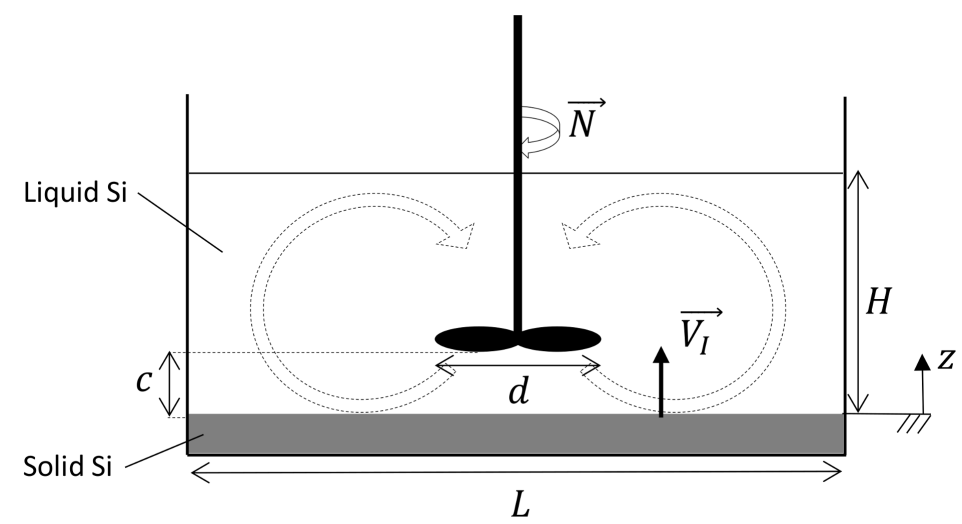

Figure 1: Mechanical stirring configuration (geometry, interface velocity $V_{I}$, rotational speed $N$ and schematic flow pattern). The silicon progressively solidifies from bottom to top. The crucible and the furnace are not presented here. The crucible has a square cross section in the $(x, y)$ plane.

It is well known that during such a process, impurities having a lower solubility in the solid phase than in the liquid, migrate from the solidification front to the liquid. It is especially the case for metallic species which have low segregation coefficients $k_{0}=C_{S}^{I} / C_{L}^{I}$, where $C_{S}^{I}$ and $C_{L}^{I}$ are the solute mass fractions at the interface on the solid side and liquid side respectively. Note that in all that follows, the subscripts and superscript $S, L, I$ and $\infty$ denote values respectively computed in the solid phase, in the liquid phase, at the interface and in the bulk of the liquid (i.e. far from the interface). The solute rejection into the liquid phase leads to the formation of a solute boundary layer which is controlled by the convection regime in the melt. A definition of the thickness of this solute boundary layer has been proposed by Wilson [43]:

$$
\delta=\frac{C_{L}^{I}-C_{L}^{\infty}}{-\left(\partial C_{L} / \partial z\right)_{I}}
$$

where $C_{L}^{\infty}$ refers to the solute mass fraction in the liquid, far from the solid/liquid interface. Using the solute conservation at the solid/liquid interface, which is defined by the following equation when diffusion is neglected in the solid:

$$
-\left.D \frac{\partial C_{L}}{\partial z}\right|_{I}=V_{I}\left(1-k_{0}\right) C_{L}^{I}
$$

with $D$ the solute diffusivity in the liquid $\left(\mathrm{m}^{2} / \mathrm{s}\right)$ and $V_{I}$ the interface velocity $(\mathrm{m} / \mathrm{s})$; a convecto-diffusive parameter $\Delta$ can be defined all along the interface by the following relation:

$$
\Delta=\frac{\delta V_{I}}{D}=\frac{C_{L}^{I}-C_{L}^{\infty}}{C_{L}^{I}\left(1-k_{0}\right)}
$$

This dimensionless boundary layer thickness defines the ratio between diffusive ( $\Delta$ tending to 1$)$ and convective $(\Delta$ tending to 0 ) transport of impurities in the melt. This parameter can then be used to define an effective segregation coefficient $k_{e f f}$, connecting the solute concentration incorporated in the solid $C_{S}^{I}$ to the bulk liquid concentration $C_{L}^{\infty}$ :

$$
k_{e f f}=\frac{C_{S}^{I}}{C_{L}^{\infty}}=\frac{k_{0}}{1-\left(1-k_{0}\right) \Delta} .
$$

As explained in former works [34, 35, 43], the analysis of the segregation problem can then be reduced to the determination of the convecto-diffusive parameter $\Delta$, which proved to be the key parameter to estimate the efficiency of a purification process by segregation.

\subsection{Hydrodynamic model}

As mentioned in the introduction, the segregation under mechanical stirring presents various time scales. In the present configuration, the crucible is not very large compared to the diameter of the impeller and its square shape is likely to have a strong influence on the fluid flow, even in the impeller blades region. The sliding mesh approach is, therefore, used to get a precise description of the impeller/crucible interaction and of the transient 
behaviour of the flow. This choice implies a thin temporal discretization for the rotation of the impeller. Then, a quasi-steady hypothesis is adopted for the solidification process, with a constant liquid height in the crucible during the simulation. The result of the simulation should be seen as a snapshot of the process for the chosen liquid height. Different liquid heights are then investigated in order to study the evolution of the flow structure during solidification $(H=20,15$ and $10 \mathrm{~cm})$. Various rotational speeds are also investigated, with $N$ ranging from 10 to $50 \mathrm{rpm}$. Forced convection is considered strong enough for natural convection to safely be neglected. In addition, the formerly mentioned plane front assumption, with constant growth rate, allows us not to have to compute the thermal problem. These simulations are performed with the commercial code Ansys Fluent ${ }^{\mathrm{TM}}$ [44]. Momentum conservation is defined by the Reynolds Averaged Navier-Stokes equations for an incompressible flow:

$$
\frac{\partial \vec{u}}{\partial t}+(\vec{u} . \nabla) \vec{u}=-\frac{1}{\rho} \nabla p+\frac{1}{\rho} \nabla\left[\left(\mu+\mu_{t}\right) \nabla \vec{u}\right]
$$

where $\vec{u}, p, \rho, \mu$ and $\mu_{t}$ stand for the fluid velocity $(\mathrm{m} / \mathrm{s})$, the pressure $(\mathrm{Pa})$, the density $\left(\mathrm{kg} / \mathrm{m}^{3}\right)$, the dynamic viscosity (Pa.s) and the turbulent viscosity $\left(\mathrm{m}^{2} / \mathrm{s}\right)$, respectively. Liquid silicon properties are used with $\rho=2550 \mathrm{~kg} / \mathrm{m}^{3}$ and $\mu=7.5 \times 10^{-4}$ Pa.s. A RANS (Reynolds Averaged Navier-Stokes) approach is used for turbulence modelling, with the realizable $k-\varepsilon$ two equations model [44]. No-slip conditions are imposed at the solidification front, at the crucible lateral walls and on the impeller surface. Regarding the free surface of the melt, a rigid-lid assumption is made and a free slip condition is applied. This assumption appears reasonable since rotational velocities remain limited, avoiding important deformations of the free surface. The deformation of the free surface was estimated a posteriori from the pressure gradients on the top surface observed in the simulations. The estimated deformation was less than $1 \mathrm{~mm}$ for configurations with $H=20$ and $15 \mathrm{~cm}$, and less than than $5 \mathrm{~mm}$ for $H=10 \mathrm{~cm}$.

The computational grid used for configurations with $H=15 \mathrm{~cm}$ is presented in figure 2. As required for the sliding mesh approach, the domain is divided in two zones delimited by a cylindrical interface. In both zones the mesh is formed of hexahedral cells, excepted in the blades region where tetrahedral cells are used because of the complex geometry. The mesh is refined in the vertical direction at the solidification front to ensure a precise description of the solute boundary layer. The first cell adjacent to the bottom surface is $5 \mu \mathrm{m}$ high and a growth rate of 1.2 is imposed.

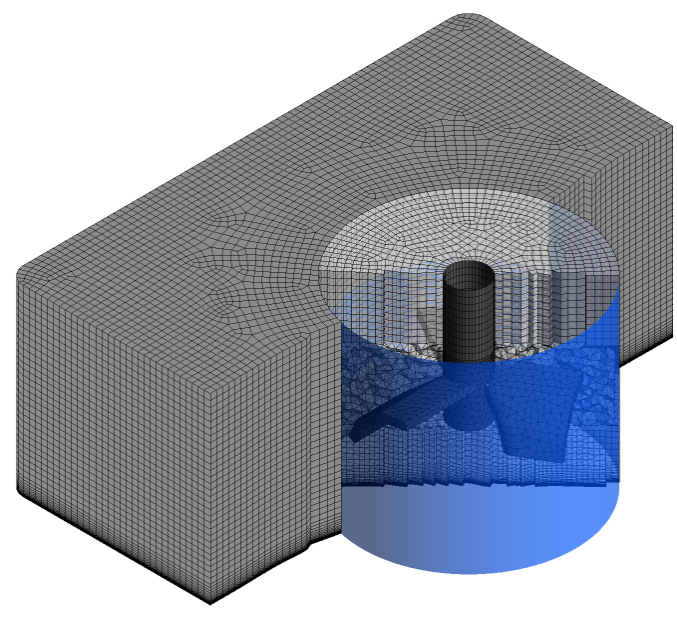

Figure 2: 3D mesh with cylindrical interface for the sliding mesh approach and solute boundary layer refinement.

\subsection{Segregation model}

The hydrodynamic simulation is coupled to a segregation computation. The solute conservation equation is solved along with the fluid flow:

$$
\frac{\partial C}{\partial t}+(\vec{u} . \nabla) C=\nabla \cdot\left[\left(D+D_{t}\right) \nabla C\right]
$$

where $C, D$ and $D_{t}$ stand for the solute mass fraction, the molecular diffusivity $\left(\mathrm{m}^{2} / \mathrm{s}\right)$ and the turbulent diffusivity $\left(\mathrm{m}^{2} / \mathrm{s}\right)$, respectively. Impurity concentrations in photovoltaic silicon are usually very low (in the order of tens of ppmw). Solute transport is, therefore, supposed to have no influence on fluid flow, which leads to a weakly coupled problem. The turbulent diffusivity is defined by a turbulent Schmidt number $S c_{t}=\nu_{t} / D_{t}$, which is fixed at 0.7 in 
the present study, based on reference studies on the subject [45, 46]. As explained in previous works [34, 41, 42, 47], solidification is modelled by a mass sink imposed in the first cells adjacent to the solidification front. Equivalent source terms are defined for the different variables of the problem [41, 42]. Solute incorporation in the solid is defined by the thermodynamic segregation coefficient $k_{0}$, leading to a solute rejection into the liquid and the formation of the solute boundary layer. Source terms are also imposed on the top surface to ensure mass and solute conservation.

\subsection{Convergence strategy}

Regarding the stirring flow, a periodic regime associated with the impeller rotation is expected. This regime should appear after an initial transient occurring when the impeller is set into motion. This initial transient is irrelevant in our configuration since stirring is initiated before the beginning of the solidification process, the silicon being completely liquid. In order to improve convergence, a steady-state MFR simulation is first performed. The segregation problem also involves an initial transient related to the formation of the solute boundary layer. The length of this initial transient depends on the solidification rate $V_{I}$, the impurity diffusivity $D$, the segregation coefficient $k_{0}$ and the convection regime [48]. To overcome this problem, segregation is first solved in a steady-state regime with the MFR flow solution. The resulting velocity and concentration fields are used as the initial solution for the transient simulation with the sliding mesh. The transient simulation is performed over several rotations of the impeller, until a periodic regime is reached. This periodic regime is identified by the temporal evolution of the flow rate in the tank and of the mean wall shear-stress on the solidification front. Once this periodic regime is reached, transient numerical results are recorded at each time-step over a complete rotation of the impeller. These results are then used to compute time averaged data and to analyse temporal fluctuations. The time-step is defined so that an elementary impeller rotation of $12^{\circ}$ is performed at each time-step.

\section{Experimental procedure}

\subsection{Experimental configuration}

An experimental characterization of the flow generated by the impeller is performed in order to confirm numerical results. This characterization consists of velocity field measurements realized by PIV on a full scale water model. For these water experiments, we assume that the hydrodynamic problem is fully characterized by the Reynolds number based on the impeller diameter $d$ and the rotation rate $N: R e_{d}=\left(N \pi d^{2}\right) /(30 \nu)$. The influence of the Froude number on the hydrodynamic regime is neglected considering that free surface deformations remain small, as explained in section 2.2. In fact, for rotational stirring systems a significant deformation of the free surface might be observed around the rotation axis due to the presence of a stationary vortex generated by the centrifugal force. But in the present configuration this phenomenon is avoided by the limited rotational speeds and the square shape of the crucible. In order to conserve the Reynolds number in the water experiments, the rotational speed is multiplied by the kinematic viscosity ratio between water and liquid silicon $\nu_{\mathrm{H}_{2} \mathrm{O}} / \nu_{\mathrm{Si}} \approx 3.4$. The investigated experimental stirring configurations are thus equivalent to numerical ones. Three liquid heights are considered $(H=10,15$ and $20 \mathrm{~cm})$. For each liquid height, three rotation speeds are tested: $N=35,70$ and $170 \mathrm{rpm}$ in water (equivalent to 10, 20 and $50 \mathrm{rpm}$ in silicon). For $H=10 \mathrm{~cm}$, the rotational speed is, however, limited to $100 \mathrm{rpm}$ (instead of $170 \mathrm{rpm}$ ) to avoid waves formation since the impeller blades are close to the free surface.

\subsection{Acquisition procedure}

For each configuration, a velocity field measurement is performed in the vertical plane normal to the lateral wall and passing through the rotation axis. This velocity field provides a good description of the flow structure in the tank. For the configurations with $H=15 \mathrm{~cm}$, supplementary measurements are performed in several vertical and horizontal planes in order to observe the three dimensionality of the flow (see figure 3). The flow is illuminated by a continuous laser and seeded with hollow glass particles, of diameter $10 \mu \mathrm{m}$, from Dantec ${ }^{\mathrm{TM}}$. Particles images are recorded thanks to a Photron ${ }^{\mathrm{TM}}$ high-speed camera, with a resolution $1024 \times 1024$ pixels. Images acquisitions are not synchronised with blades rotation. The time interval between 2 successive frames is comprised between $2 \mathrm{~ms}$ and $12 \mathrm{~ms}$, depending on the impeller rotational speed and the position of the observation plane. The mean velocity field is obtained by averaging results acquired over several complete rotations (17 rotations at least). Mean convergence is analysed to ensure that a sufficient number of instantaneous velocity fields is considered. This averaging procedure filters turbulence fluctuations and the transient behaviour of the flow inherent to the rotation of the impeller. 



Figure 3: Locations of the horizontal and vertical measurement planes used for configurations with $H=15 \mathrm{~cm}$. The present paper focuses on the results obtained for the central vertical plane (at $y=0)$ and for the first horizontal plane $($ at $z=1 \mathrm{~cm})$.

\subsection{PIV computation}

Velocity fields are obtained from particle images thanks to the PIV algorithm DPIVsoft, developed by the IRPHE laboratory under Matlab ${ }^{\mathrm{TM}}$ [49]. It is based on a cross-correlation computation by Fast Fourier Transform. As explained by Meunier and Leweke [50], this algorithm is optimized for high velocity gradients flows. An iterative procedure with correlation windows translation and deformation is used to reduce displacements computation errors related to particles loss and correlation patterns deformation. This procedure is much valuable for the studied configuration since the impeller flow is expected to feature high velocity gradients.

Moreover, the axial impeller generates a fully 3D flow, implying significant out of plane particles motion. This out of plane motion must be considered for the definition of the time interval between the frames used for the correlation computation. The time interval must be carefully chosen in order to achieve measurable particles displacements and limit particles loss leading to aberrant vectors. The use of a continuous laser and a fast camera with a constant acquisition frequency makes this aspect easier to handle. Indeed, the correlation computation can be performed on directly consecutive images, or between images separated by a certain number of intermediate frames. In this work, the appropriate time interval is estimated from the out of plane velocity observed in the numerical simulations. After the PIV computation, a median criteria is used to detect and replace some of the remaining aberrant vectors.

Uncertainties related to the PIV computation depend, among others, on the magnitude of the measured displacement and the shear rate in the flow. Meunier and Leweke [50] tested the PIV algorithm thanks to artificial particles images with an imposed displacement and a unidirectional shear. For the present study, equivalent tests were performed, but with a rotation condition instead of a unidirectional shear. The aim is to estimate measurement uncertainties in our experiments. Similar RMS errors are observed in our tests and in authors original publications $[49,50]$. Considering the mean displacements and shear rates observed in our measurements, the uncertainty on the measured displacement appears to be in the order of \pm 0.12 pixels. Global relative uncertainties are then obtained from this uncertainty estimate and the mean displacements observed in the different measurements. For the different measurements, these global uncertainties remain below $\pm 10 \%$, excepted for three configurations where the out of plane motion is too important to achieve a mean displacement larger than 1 pixel without producing a large number of aberrant vectors. On the contrary, on horizontal planes close to the bottom wall $(z=1 \mathrm{~cm}$ or $z=2 \mathrm{~cm})$ the flow is almost $2 \mathrm{D}$ and global uncertainties remain in the order of $\pm 5 \%$ since a larger particles mean displacement is achieved. Clearly, this analysis only provides a rough estimate of the measurement uncertainties, on the basis of averaged values. The precise determination of PIV uncertainties is a wide and complex subject which is beyond the scope of the present work. The elements presented above give however a good estimate of the degree of confidence that can be placed in our mean velocity field measurements.

\section{Results}

Different stirring configurations are investigated, both numerically and experimentally. Three liquid heights $H$ are tested in order to analyse flow structures corresponding to different instants of the solidification process. The highest liquid height, $H=20 \mathrm{~cm}$, corresponds to the beginning of the solidification for a pilot scale process. For the smallest liquid height, $H=10 \mathrm{~cm}$, the impeller is close to the free surface of the melt since the distance from the bottom of the impeller to the front is kept at $c=5 \mathrm{~cm}$. Smaller liquid heights would lead to a close proximity 
of impeller blades with the solidification front and/or the liquid free surface. Such critical configurations are not investigated in this study because they can be considered as unacceptable for a well controlled industrial process. An intermediate height, $H=15 \mathrm{~cm}$, is also considered. For these three liquid heights, three rotational speeds are considered with $N=10,20$ and $50 \mathrm{rpm}$ in silicon. The corresponding Reynolds numbers are $\operatorname{Re}_{d}=6.0 \times 10^{4}$, $1.2 \times 10^{5}$ and $3.0 \times 10^{5}$. This range of parameters might appear somehow limited, but our objective is to present a methodology for the study of segregation in realistic configurations regarding process requirements.

Regarding the solute segregation computation coupled with the hydrodynamic simulations, we define a solidification front velocity $V_{I}=10^{-5} \mathrm{~m} / \mathrm{s}$, a solute diffusivity $D=10^{-8} \mathrm{~m}^{2} / \mathrm{s}$ and a thermodynamic segregation coefficient $k_{0}=0.3$. At the initialization, the solute mass fraction in the liquid is uniform and equal to $C_{0}=20 \mathrm{ppmw}$ (part per million weight). Numerical concentrations are used to compute the convecto-diffusive parameter $\Delta$ from equation (3). $\Delta$ is computed locally at each point of the solidification front and at each time-step of the transient simulations. The spatial variations of $\Delta$ provide information regarding lateral segregations and the surface averaged value $\langle\Delta\rangle$ describes the axial segregation regime.

\subsection{Time averaged flow}

Numerically obtained velocity fields in the central vertical plane for the different liquid heights are presented on the left hand side of the figure 4 , for the rotational speed $N=20 \mathrm{rpm}$. These velocity fields are averaged over a complete rotation of the impeller. Numerical results are compared to experimental ones thanks to the PIV velocity fields obtained for equivalent configurations on the right hand side of the figure 4. For this comparison, velocity are normalized by the blade tip velocity $V_{\text {tip }}=(N \pi d) / 60(\mathrm{~m} / \mathrm{s})$. For $H=20 \mathrm{~cm}$ (figures $4(\mathrm{a})$ and $\left.4(\mathrm{~b})\right)$ and $H=15 \mathrm{~cm}$ (figures 4(c) and 4(d)), an axial downward flow is observed, as expected for the chosen impeller geometry. This flow impinges the solidification front and leads to a parallel flow over the major part of the front. A main recirculation develops in the crucible and secondary vortices are observed under the rotation axis. From these results, we can see that the impeller has a direct influence on convection conditions above the solidification front. The observed high velocities in the jet suggest an efficient action on the solute mass transfer. For these axial flow configurations, a good agreement is found between transient numerical simulations and PIV measurements. The global structure of the velocity field is well retrieved in the simulations and numerical velocities in the jet are consistent with experimental ones. The parallel flow over the front can be observed on the velocity fields obtained in the horizontal plane $z=1 \mathrm{~cm}$, presented in figure 5 for $H=15 \mathrm{~cm}$. Here again, the agreement between simulations and PIV measurements is quite satisfying. Directly below the impeller, a tangential flow is observed. Beyond the impeller radius, the parallel flow generated by the jet arises and the radial velocity component prevails. Close to lateral walls, the velocity magnitude is reduced and vortices are observed in the corners. For $H=10 \mathrm{~cm}$ (figures $4(\mathrm{e})$ and $4(\mathrm{f})$ ) a radial flow is observed. The reduction of the liquid height above the impeller limits the pumping effect required for the formation of an axial jet. Velocities projections in the vertical plane present lower amplitudes in comparison to axial flow configurations. This behaviour is also observed experimentally which confirms that different hydrodynamic regimes occur during the solidification process. Several segregation regimes are thus to be expected. For these radial flows, simulations underestimate fluid velocities in the main recirculation compared to experimental results.

For a given liquid height, the rotational speed is found to have little influence on the global flow structure. As an example, figure 6 presents vertical profiles of the in-plane velocity components $u$ and $w$, taken at $x=0.1 \mathrm{~m}$. These profiles are extracted from numerical and experimental results for $H=15 \mathrm{~cm}$ and three rotational speeds. In the numerical results (figures 6(a) and 6(c)), normalized velocity profiles appear independent of the rotational speed. Experimental profiles of the horizontal component $u$ (figure 6(b)) confirm this point. For the vertical component $w$, the agreement between numerical and experimental results is less satisfying. In fact, PIV measurements show an increase in the maximum normalized velocity $w / V_{t i p}$ when the rotational speed is increased (figure $6(\mathrm{~d})$ ), which is not retrieved in numerical results. 


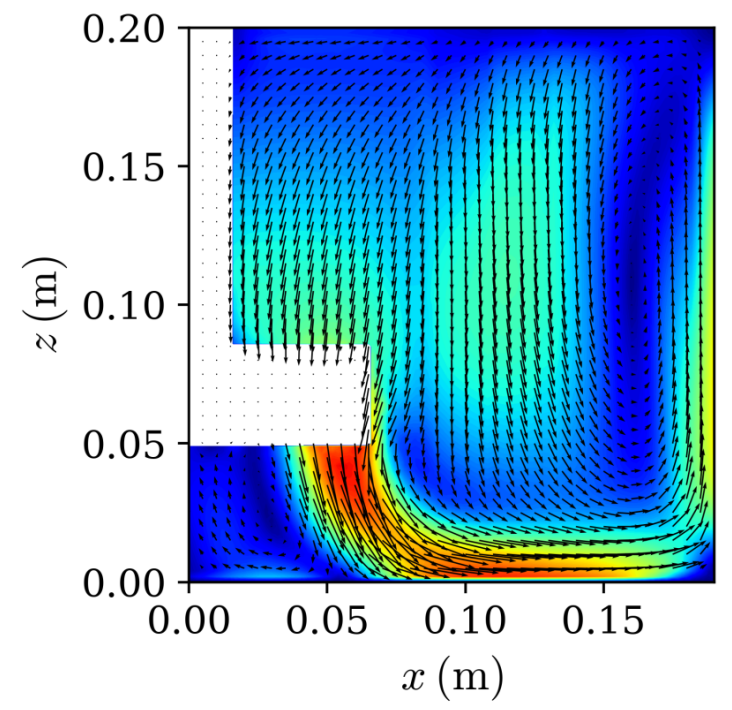

(a) simulation $H=20 \mathrm{~cm}$

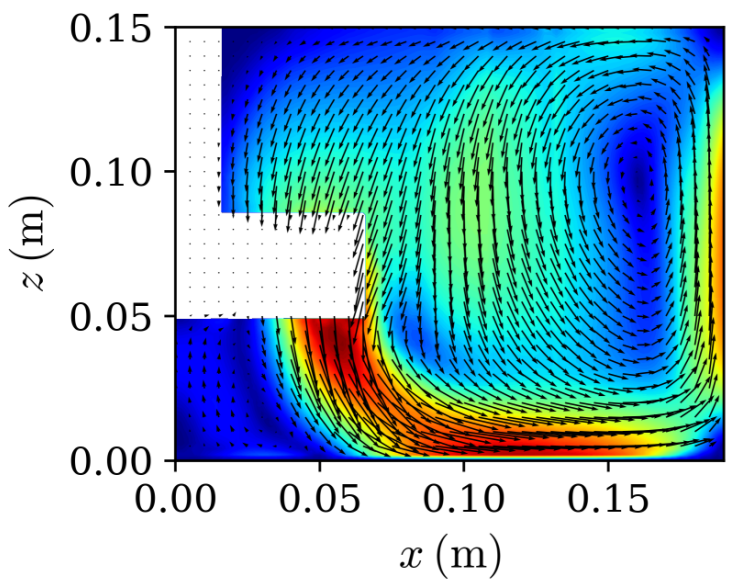

(c) simulation $H=15 \mathrm{~cm}$

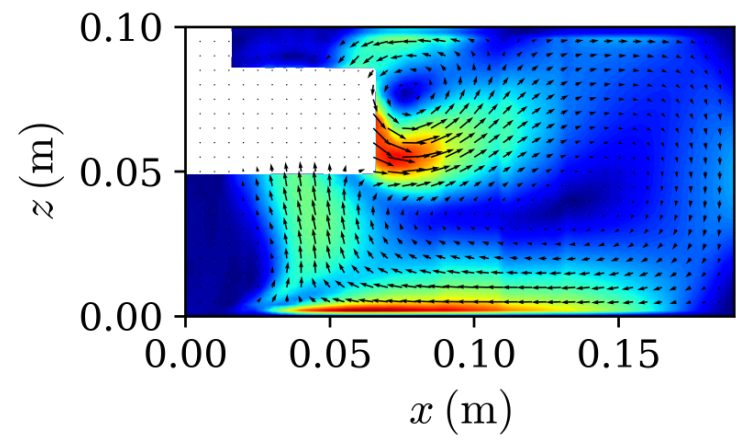

(e) simulation $H=10 \mathrm{~cm}$



(b) experiment $H=20 \mathrm{~cm}$

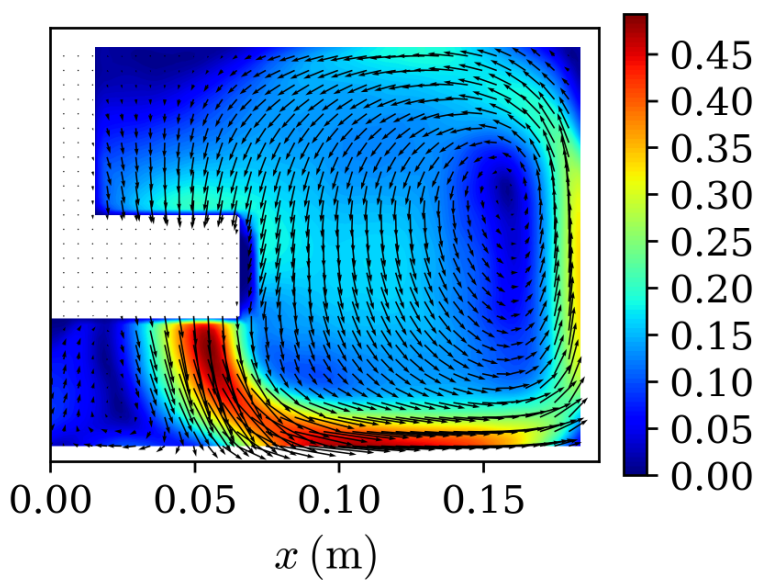

(d) experiment $H=15 \mathrm{~cm}$

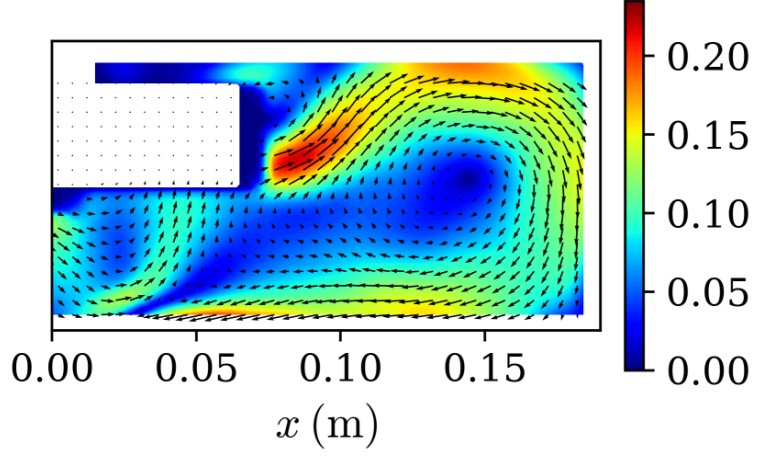

(f) experiment $H=10 \mathrm{~cm}$

Figure 4: Time-averaged velocity fields in the vertical middle plane $(y=0 \mathrm{~cm})$, for $N=20 \mathrm{rpm}$ in silicon (i.e. $N=70 \mathrm{rpm}$ in the water model). Colormaps represent the magnitude of the in-plane velocity component for different liquid heights. These magnitudes are normalised by the blade tip velocity $V_{t i p}$. Only the right side of the plane is presented $(x \geq 0)$. The impeller region and the locations where valid velocity measurements are not available are represented in white. 


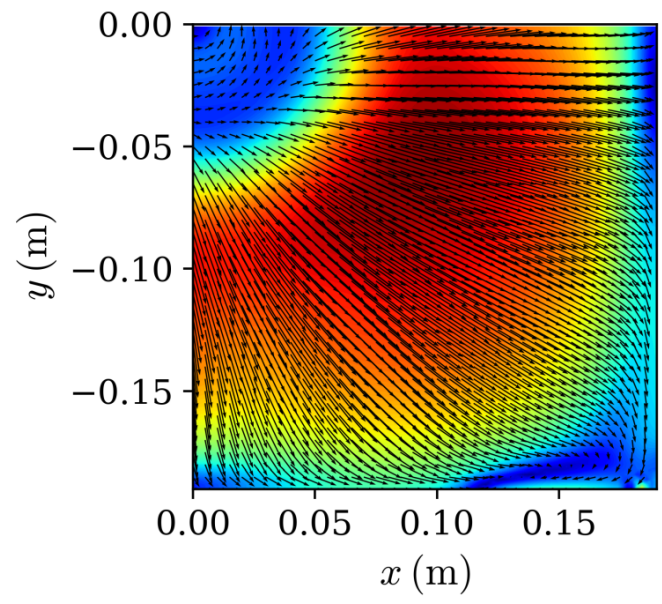

(a) simulation



(b) experiment

Figure 5: Time-averaged velocity fields in a horizontal plane $1 \mathrm{~cm}$ above the solidification front $(z=1 \mathrm{~cm})$, for $H=15 \mathrm{~cm}$ and $N=20 \mathrm{rpm}$ in silicon (i.e. $N=70 \mathrm{rpm}$ in the water model). Colormaps represent the magnitude of the in-plane velocity component. These magnitudes are normalised by the blade tip velocity $V_{t i p}$. Only one quarter of the plane is presented and the rotation axis is located at the top left corner. 


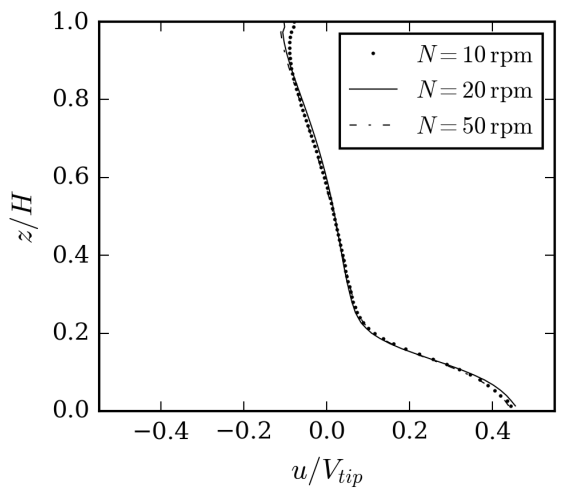

(a) $x$ velocity component, numerical

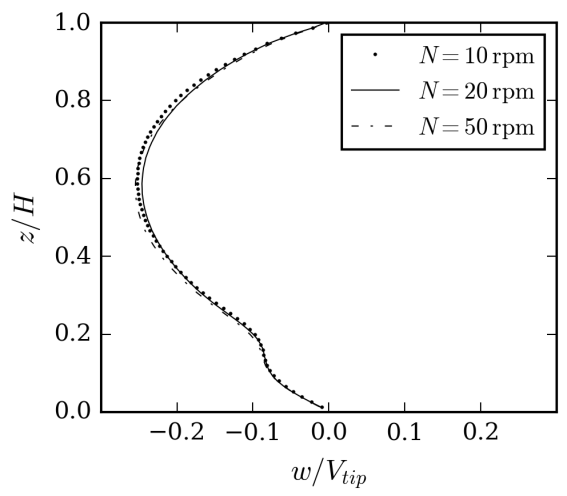

(c) $z$ velocity component, numerical

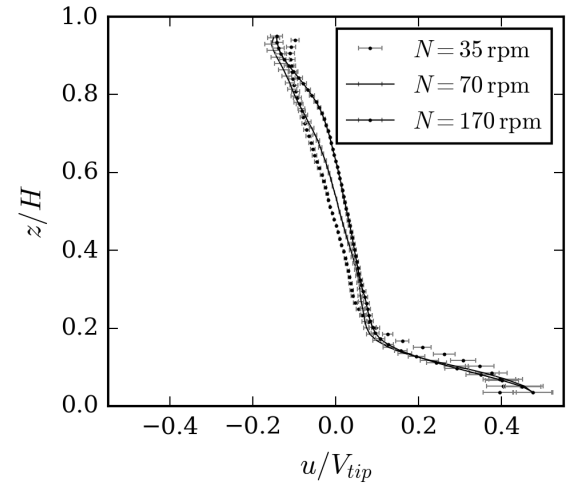

(b) $x$ velocity component, experimental

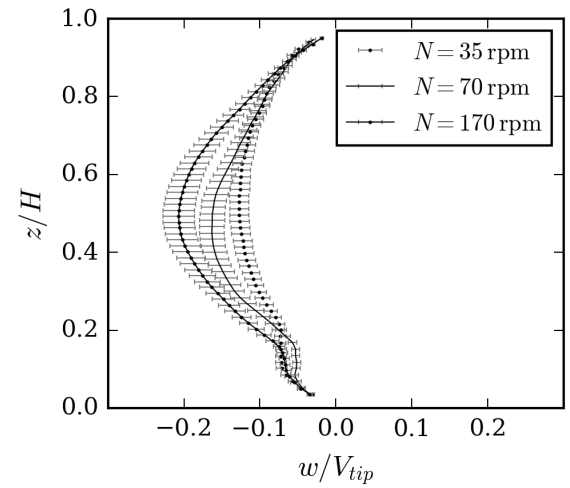

(d) $z$ velocity component, experimental

Figure 6: Time-averaged numerical and experimental velocity profiles taken at $x=0.1 \mathrm{~m}$, for $H=15 \mathrm{~cm}$. Velocities are normalised by the blade tip velocity $V_{\text {tip }}$. The error bars in figures $6(\mathrm{~b})$ and $6(\mathrm{~d})$ represent the uncertainty of $\pm 10 \%$ estimated for the PIV computation (see section 3.3).

\subsection{Unsteady features of the flow}

The previous results were obtained from a time average of the flow over a complete rotation of the impeller. The impeller rotation induces, however, some periodic fluctuations. First, for axial flow configurations $(H=20 \mathrm{~cm}$ and $15 \mathrm{~cm}$ ), detached eddies can be observed in the transient simulations results. These detached eddies appear at each passage of the blades and are transported in the axial jet. Such eddies can be seen in the instantaneous velocity and vorticity fields presented in figure 7. For the chosen impeller angular position, a detached eddy is observed in the left part of the velocity field (figure $7(\mathrm{a})$ ), between $x=-10 \mathrm{~cm}$ and $-5 \mathrm{~cm}$. This eddy is depicted on the vorticity field (figure $7(\mathrm{~b})$ ) by a spot of high positive vorticity. Other spots are also visible in both right and left side of the rotation axis, further in the impeller jet. These spots denote previous detached eddies, advected in the jet and progressively dissipated. These large transient structures can have a significant influence on the solute transport, justifying the use of a sliding mesh approach for the description of the fluid flow dynamics. 


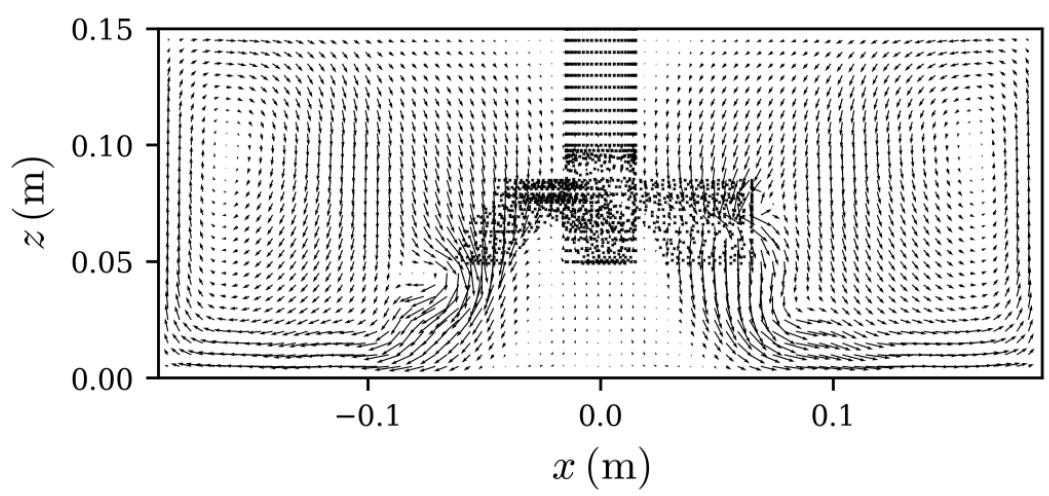

(a) instantaneous velocity field



(b) instantaneous vorticity field

Figure 7: Instantaneous numerical velocity $(7(\mathrm{a}))$ and vorticity fields $(7(\mathrm{~b}))$ in the central vertical plane $y=0 \mathrm{~cm}$, for $H=15 \mathrm{~cm}$ and $N=20 \mathrm{rpm}$. The colormap represents the normal vorticity component $\omega_{y}$ normalized by the impeller rotation frequency $(N \pi) / 30$ $(\mathrm{rad} / \mathrm{s})$.

Moreover, the wall shear-stress repartition on the front exhibits temporal fluctuations associated with the rotation of the impeller. We focus here on the interface shear-stress because it proved to be a meaningful indicator of the convection regime in the solute boundary-layer $[34,37,47]$. The temporal fluctuations can be observed on figure 8(a) which presents 10 instantaneous shear-stress profiles recorded at successive time-steps during the transient simulation for the configuration $H=15 \mathrm{~cm}$ and $N=20 \mathrm{rpm}$. Each profile then corresponds to a different angular position of the impeller. The shear-stress profile corresponding to the time-averaged solution over a complete rotation of the impeller is also presented. Significant temporal fluctuations of the local shear-stress can be observed around the time averaged results, notably in the region of the jet generated by the impeller $(r>d / 2)$. Such fluctuations question the validity of the quasi-steady assumption used for the segregation problem. It can however be argued that, in this case, the time scale representative of the convection fluctuations is small compared to the time scale of the diffusion at the scale of the solute boundary layer. To compare these time scales, a dimensionless pulsation $F q[51,52]$ can be defined by the following relation:

$$
F q=\left(3 N \frac{\pi}{30}\right) \frac{\langle\bar{\delta}\rangle^{2}}{D}
$$

where $(3 N \pi) / 30$ represents the pulsation of the perturbation, with 3 the number of blades; and $\langle\bar{\delta}\rangle$ stands for the time and space average of the solute boundary layer thickness. Dimensionless pulsations $F q$ are presented in table 1 for the different flow configurations. $F q$ remains larger than 1 for all tested configurations. As explained by Garandet [51], $F q>1$ corresponds to a high frequency regime. In this case convection fluctuations are to fast for the solute boundary layer to adapt and concentration fluctuations are filtered.

This point can be addressed in a more pragmatic way by comparing the fluctuations of the wall shear-stress $\tau$ and the fluctuations of the convecto-diffusive parameter $\Delta$. For this purpose, instantaneous profiles of $\Delta$ are presented 
in figure $8(\mathrm{~b})$ for the configuration $H=15 \mathrm{~cm}$ and $N=20 \mathrm{rpm}$. No temporal fluctuations can be identified from these instantaneous profiles. In order to quantify the shear-stress fluctuations, we define a local fluctuation rate $\tau_{v a r}$ at each point along the diagonal by the following relation:

$$
\tau_{v a r}(r)=\max (|\tau(r, t)-\overline{\tau(r)}|) / \overline{\tau(r)}
$$

where $\tau(r, t)$ and $\overline{\tau(r)}$ stand for the instantaneous local value of shear-stress and the time-averaged value observed during a complete rotation, respectively. Averaged values of these fluctuation rates along the diagonal, denoted $\left\langle\tau_{v a r}\right\rangle$, are presented in table 1 for the different stirring configurations. The most important fluctuations are observed for axial flow configurations $(H=15 \mathrm{~cm}$ and $20 \mathrm{~cm})$, with an averaged variation rate up to $12.9 \%$. For radial flow configurations $(H=10 \mathrm{~cm})$, fluctuations are less pronounced with a mean fluctuation rate below $3 \%$. On the other hand, the mean fluctuation rate of the convecto-diffusive parameter, $\left\langle\Delta_{v a r}\right\rangle$, remains below $1.24 \%$ for all tested configurations. As expected, convection fluctuations appear to have a very limited influence on the solute boundary layer, which only depends on the time averaged flow. We thus consider that a quasi-steady approach is able to give a good estimate of the segregation regime for this forced convection configuration.

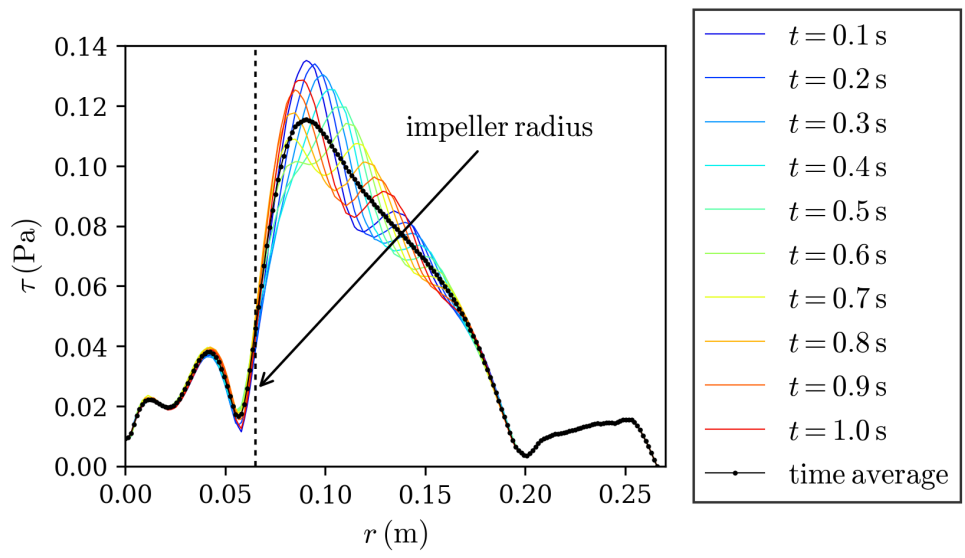

(a) $\tau$ profiles

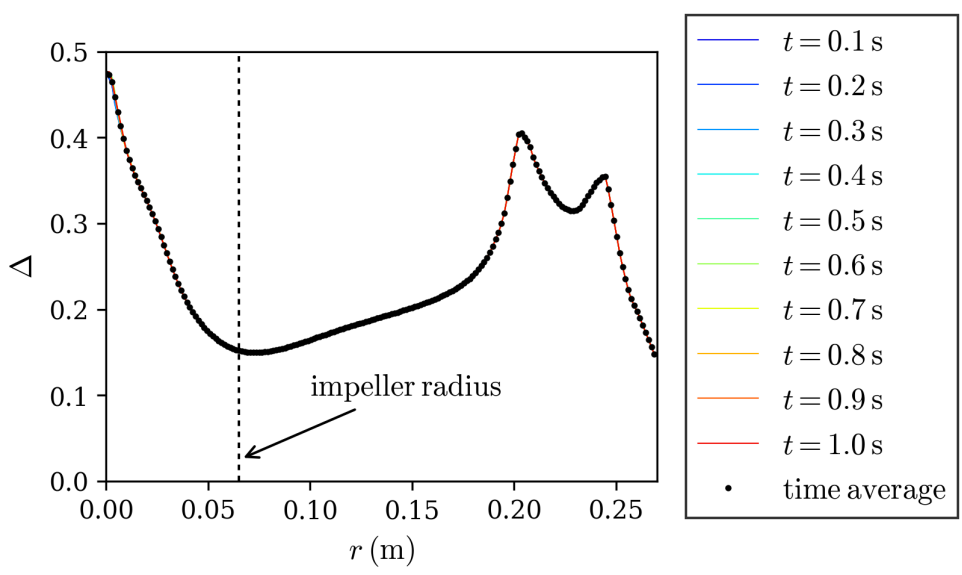

(b) $\Delta$ profiles

Figure 8: Shear-stress $\tau$ and convecto-diffusive parameter $\Delta$ instantaneous profiles for ten successive angular positions of the impeller blades and time averaged profiles, for the configuration $H=15 \mathrm{~cm}$ and $N=20 \mathrm{rpm}$. The abscissa $r$ represents the distance from the rotation axis along the diagonal of the crucible. The convecto-diffusive parameter is computed from equation (3) with $V_{I}=10^{-5} \mathrm{~m} / \mathrm{s}$ and $D=10^{-8} \mathrm{~m}^{2} / \mathrm{s}$. 
Table 1: Numerically obtained spatial and temporal average, $\langle\bar{\Delta}\rangle$, of $\Delta$ over the solid/liquid interface and a complete rotation of the impeller computed from equation (3); dimensionless pulsations $F q$ computed from equation (7); and averaged fluctuation rates, $\left\langle\tau_{v a r}\right\rangle$ and $\left\langle\Delta_{v a r}\right\rangle$, for $\tau$ and $\Delta$.

\begin{tabular}{cccccc}
\hline$H(\mathrm{~cm})$ & $N(\mathrm{rpm})$ & $\langle\bar{\Delta}\rangle$ & $F q$ & $\left\langle\tau_{\text {var }}\right\rangle$ & $\left\langle\Delta_{\text {var }}\right\rangle$ \\
\hline 20 & 10 & 0.338 & 36 & $8.16 \%$ & $0.62 \%$ \\
20 & 20 & 0.237 & 35 & $11.0 \%$ & $1.04 \%$ \\
20 & 50 & 0.127 & 25 & $11.5 \%$ & $1.24 \%$ \\
15 & 10 & 0.339 & 36 & $7.15 \%$ & $0.22 \%$ \\
15 & 20 & 0.233 & 34 & $9.42 \%$ & $0.31 \%$ \\
15 & 50 & 0.123 & 24 & $12.9 \%$ & $1.16 \%$ \\
10 & 10 & 0.481 & 73 & $1.87 \%$ & $0.08 \%$ \\
10 & 20 & 0.333 & 69 & $2.22 \%$ & $0.16 \%$ \\
10 & 50 & 0.205 & 66 & $2.83 \%$ & $0.26 \%$ \\
10 & 30 & 0.271 & 69 & $2.41 \%$ & $0.15 \%$ \\
\hline
\end{tabular}

\subsection{Segregation quasi-steady simulations}

The influence of the stirring parameters on the segregation process are now estimated thanks to the numerical simulations. Figure 9 presents the repartition of the convecto-diffusive parameter $\Delta$ on the solidification front for $H=15 \mathrm{~cm}$ and $H=10 \mathrm{~cm}$, with $N=20 \mathrm{rpm}$; and figure 10 presents $\Delta$ profiles along the diagonal for the different liquid heights and rotational speeds. Regarding a purification process by directional solidification, a first issue is to achieve an efficient segregation in the major part of the ingot in order to maximize the material yield. For axial flow configurations, figure 9(a) shows that an efficient segregation, i.e. low values of $\Delta$, can be achieved on the major part of the front, where the parallel flow generated by the impeller jet occurs. But higher values of $\Delta$ are observed near lateral walls, corners and below the rotation shaft. This repartition is directly correlated to the flow structure in the crucible since convection is less intense in these locations. When the liquid height becomes small, the transition to a radial flow leads to a less efficient segregation, as shown by the mean values $\langle\bar{\Delta}\rangle$ reported in table 1 . In addition, a more complex solute repartition can be observed on the solidification front. Figure 9 (b) clearly shows that, even close to the impeller region, high values of $\Delta$ can be expected for radial flows. These spatial variations represent a drawback for a purification process since concentration variations in the solidified ingot must be limited. An interesting result is that the increase of the rotational speed $N$ reduces $\Delta$ on the complete solid/liquid interface, even in the corners and under the rotation shaft, as observed in figure 10. This trend is observed for both axial and radial flows.

These results confirm that efficient stirring conditions can be obtained with a mechanical impeller. The purification process can be optimized in two ways. First, a modification of the impeller design could reduce the spatial variations of $\Delta$. Second, a modulation of the rotational speed could be applied in order to compensate the reduction of the stirring efficiency when a radial flow appears. 


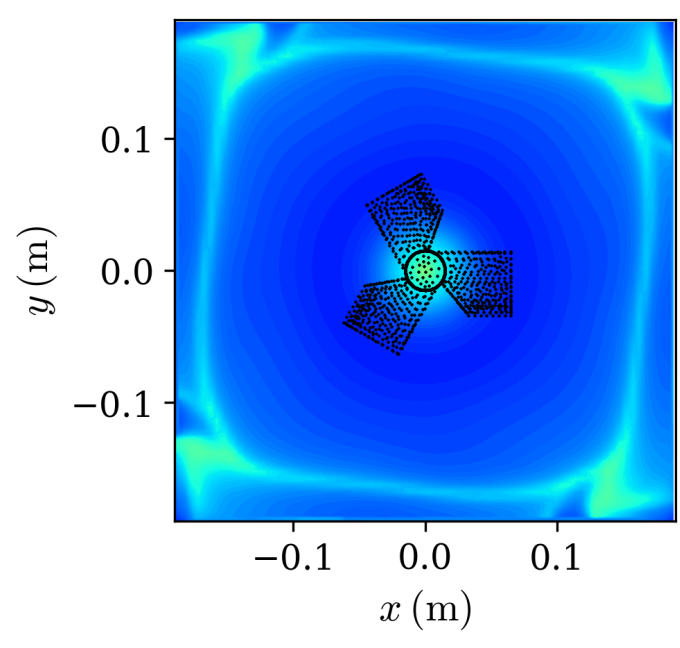

(a) $H=15 \mathrm{~cm}$

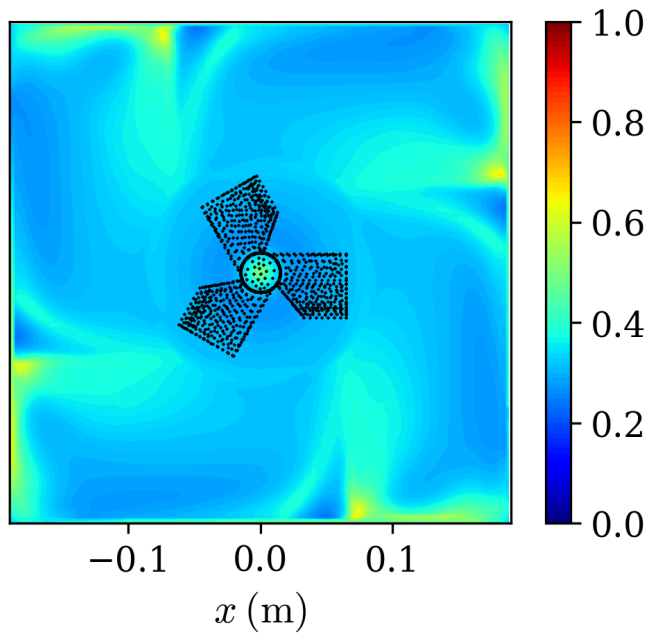

(b) $H=10 \mathrm{~cm}$

Figure 9: Repartition of the convecto-diffusive parameter $\Delta$ on the solidification front, for the rotational speed $N=20 \mathrm{rpm}$ and two different liquid heights: $H=15 \mathrm{~cm}(\mathrm{a})$ and $H=10 \mathrm{~cm}(\mathrm{~b})$.

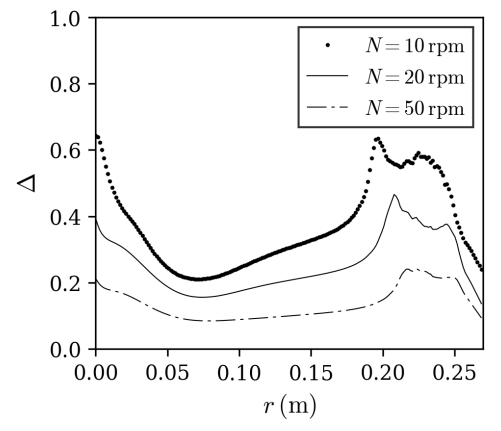

(a) $H=20 \mathrm{~cm}$

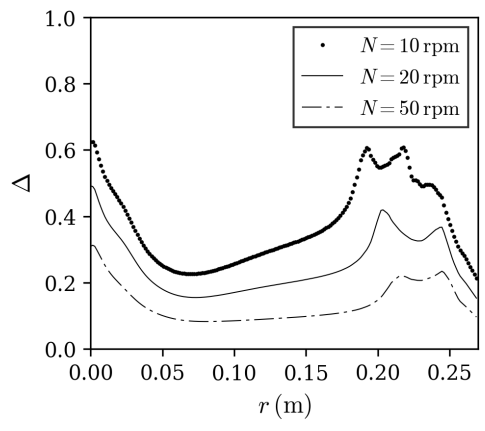

(b) $H=15 \mathrm{~cm}$

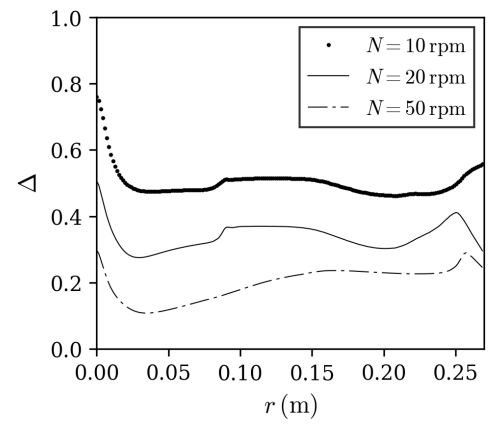

(c) $H=10 \mathrm{~cm}$

Figure 10: Profiles of the convecto-diffusive parameter $\Delta$ on the solidification front along a diagonal for different liquid heights $H$ and rotational speeds $N$.

\subsection{Segregation analytical approach}

The numerical segregation results are now compared to an analytical approach based on the combination of hydrodynamic simulations and a scaling analysis of the solute boundary layer thickness. In their original publication, Garandet et al. [34] provide an analytical expression of the convecto-diffusive parameter $\Delta$ as a function of a unique dimensionless parameter $B=\tau D^{2} /\left(V_{I}^{3} \mu l\right)$. This expression is obtained from a scaling analysis procedure, considering that a boundary layer flow develops over the solidification front. The wall shear-stress at the solidification front, $\tau$, is used as an indicator of the convection intensity in the solute boundary layer. The characteristic length $l$ represents the distance over which the boundary layer flow takes place, until separation. This analytical model has recently been improved to account for turbulence diffusivity effects, using the well-known mixing length model to describe the turbulent diffusivity profile inside the solute boundary layer [36]. Readers are referred to the original publication [36] for the complete derivation of the model. This analysis leads to the definition of a critical value $B_{c}$ beyond which turbulent transfer must be taken into account in the solute boundary layer. This critical value is defined as a function of a dimensionless parameter $\psi=\left(\kappa / c_{1}\right) \sqrt{S c / S c_{t}} R e_{V_{I}}$ by the following relation:

$$
B_{c}=\frac{1}{\psi}\left(1+\frac{1}{\psi}\right)^{2}
$$


where $\kappa=0.4$ and $c_{1}=26$ are empirical constants of the turbulence model, $S c=\nu / D$ is the Schmidt number and $\operatorname{Re}_{V_{I}}=\left(V_{I} l\right) / \nu$ is a Reynolds number based on the interface velocity. For moderate regimes the original model proposed by Garandet et al. [34] is valid, and the convecto-diffusive parameter is expressed by the following relation:

$$
\Delta=(2 B)^{-1 / 3}\left\{\left(1+\sqrt{1+\frac{4}{27 B}}\right)^{1 / 3}+\left(1-\sqrt{1+\frac{4}{27 B}}\right)^{1 / 3}\right\}, \quad \text { for } B<B_{c} .
$$

For turbulent convective regimes, with $B \geq B_{c}$, the convecto-diffusive parameter can be estimated from an asymptotic regime defined by the following relation:

$$
\Delta=(\psi B)^{-1 / 2}, \quad \text { for } B>>B_{c} .
$$

These studies $[34,36]$ are dedicated to a canonical 2D lid driven cavity configuration which is used as a reference test case for the validation of the segregation analytical model. Our aim is now to apply this model to a more realistic configuration, namely a square cross section crucible with forced convection induced by a mechanical stirrer. In order to compare this analytical model to our numerical results, mean values of shear-stress, observed in the simulations, are used to compute the mean segregation parameter $\langle\bar{B}\rangle$; and mean values of the convecto-diffusive parameter, $\langle\bar{\Delta}\rangle$, are directly extracted from numerical results for each rotational speed and liquid height. In a first attempt, and based on the flow structures observed in section 4.1, the characteristic length $l$ is here equated to the half width of the crucible $L / 2=0.19 \mathrm{~m}$. Results are presented in figure 11. For comparison purposes, numerical results obtained for the 2D transient lid driven cavity configuration are also presented [36]. In both cases the same parameters were used for the interface velocity $V_{I}$ and the solute diffusivity $D$. For the lid driven cavity, the characteristic length $l$ is defined by the cavity width $L_{2 D}=0.2 \mathrm{~m}$. Equation (9) provides a critical value $B_{c} \approx 9$ for the $2 \mathrm{D}$ configuration and $B_{c} \approx 10$ for the 3D configuration with mechanical stirring. The influence of the turbulent diffusivity in the solute boundary layer is thus expected to appear for similar hydrodynamic regimes. As can be seen in figure 11, it is quite remarkable that 3D numerical results follow the same law than $2 \mathrm{D}$ results obtained with a different flow configuration. It confirms that the parameter $B$ is able to capture the physics of the segregation phenomena through equations (9), (10) and (11), independently of the convection driving force. An additional simulation has been performed with $N=100 \mathrm{rpm}$, in order to confirm the trend for highly convective regimes. The introduction of turbulent transport is justified since all tested configurations are close to, or overcome, the critical value $B_{c}$. Relation (11) provides a good estimate of the solute boundary layer thickness for the studied configurations from pure scaling arguments, and without fitting parameter. As formerly shown in the case of the 2D lid driven cavity, an hydrodynamic simulation of the stirring flow, without solute transport resolution and solute boundary layer refinement, would have then been sufficient to determine the mean segregation regime. It must be noted that the analytical model is based on quasi-steady assumption. Fluctuations analysis (similar to the one presented in section 4.2) is required to verify the validity of the model and to determine if instantaneous or time-averaged wall shear-stress values must be used as input data for the estimation of the segregation regime.

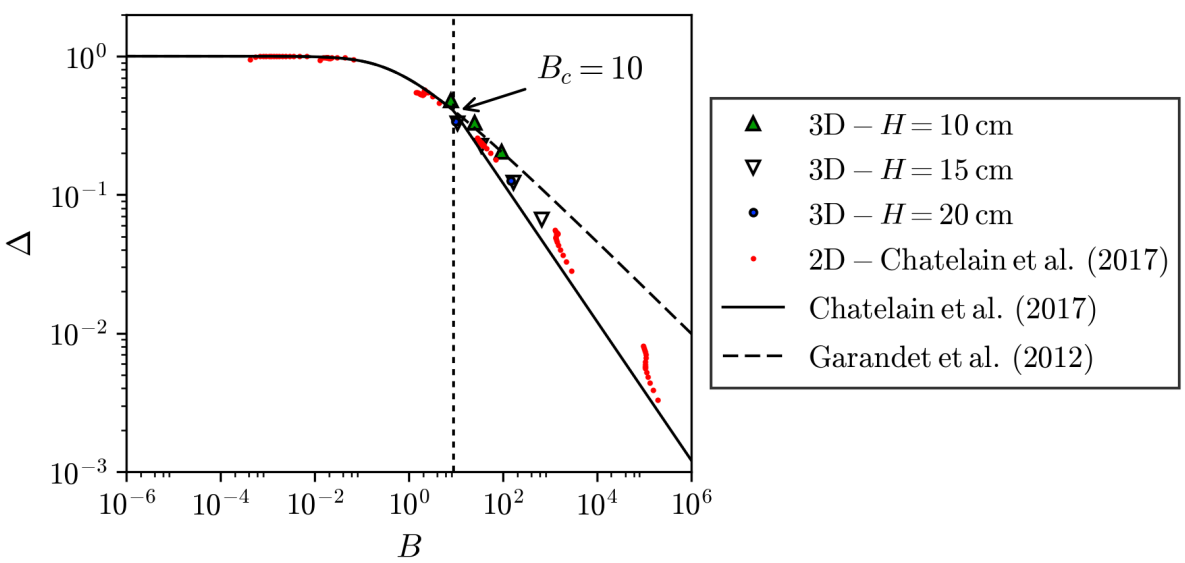

Figure 11: Mean convecto-diffusive parameter $\langle\bar{\Delta}\rangle$ as a function of the mean segregation parameter $\langle\bar{B}\rangle$. Comparison between our 3D numerical results with $N=10,20,50$ and $100 \mathrm{rpm}$ and three different liquid heights $H$; $2 \mathrm{D}$ transient numerical results for a lid driven cavity configuration [36] and analytical models (relations (10) and (11)) 


\section{Conclusions}

Numerical simulations of solute segregation under mechanical stirring in a square cross section crucible have been performed. The purpose of the developed numerical model is to determine the efficiency of a silicon purification process by directional solidification. The ability of the numerical model to reproduce the hydrodynamics has been validated by comparison with a scaled water experiment. The efficiency of the process is expressed in terms of convecto-diffusive parameter repartition on the solidification front. Meaningful information is then extracted from the simulations regarding the mean segregation regime, the lateral segregations and the evolution of the stirring efficiency during the solidification process. Low values of convecto-diffusive parameter, meaning efficient segregation, can be achieved even in corners and underneath the impeller. In our opinion, this kind of model could be used for optimization studies regarding process parameters and impeller design, in order to maximize the material yield of the purification process. The relevance of the model could, obviously, be increased by taking into account the solidification front curvature, as demonstrated by Dumitrica et al. [12]. On the other hand, the precise hydrodynamic results could be used to estimate the influence of the stirring system on the thermal conditions of the process.

The numerical segregation results are also compared to an analytical model, derived from a scaling analysis of the solute boundary layer. It appears that this approach provides a good estimate of the mean segregation regime, especially when the turbulent transport in the solute boundary layer is taken into account. This is a generalisation of the results previously obtained for the canonical 2D lid driven cavity configuration. This approach offers an opportunity to simplify the simulations, reducing the problem to the resolution of the fluid flow, and avoiding the numerical resolution of the solute boundary layer. Nevertheless, before using this method to analyse lateral segregations and determine segregation patterns in solidified ingots, as proposed in a recent publication [36]; important issues must be treated. We can for instance mention the analysis of the 3D flow structure, for the definition of the different recirculations sizes and the detection of the separation and impingement points, where the analytical model must be regularized.

\section{Acknowledgements}

The authors are indebted to the institute Carnot Ingnierie@Lyon for its support and funding. We also warmly thank J.P. Garandet and C. Audoin from the CEA and D. Henry from the LMFA for many fruitful discussions on the subject.

\section{References}

[1] J.R. Davis, A. Rohatgi, R.H. Hopkins, P.D. Blais, P. Rai-Choudhury, J.R. McCormick, and H.C. Mollenkopf. Impurities in silicon solar cells. IEEE Transactions on Electron Devices, 27:677-687, 1980. ISSN 0018-9383. DOI 10.1109/T-ED.1980.19922.

[2] J. Hofstetter, J.F. Lelièvre, C. del Canizo, and A. Luque. Acceptable contamination levels in solar grade silicon: From feedstock to solar cell. Materials Science and Engineering: B, 159-160:299-304, 2009. ISSN 09215107. DOI 10.1016/j.mseb.2008.05.021.

[3] Y. Delannoy. Purification of silicon for photovoltaic applications. Journal of Crystal Growth, 360:61-67, 2012. ISSN 00220248. DOI 10.1016/j.jcrysgro.2011.12.006.

[4] F. A. Trumbore. Solid Solubilities of Impurity Elements in Germanium and Silicon*. Bell System Technical Journal, 39:205-233, 1960. ISSN 00058580. DOI 10.1002/j.1538-7305.1960.tb03928.x.

[5] R.W. Series and D.T.J. Hurle. The use of magnetic fields in semiconductor crystal growth. Journal of Crystal Growth, 113:305-328, 1991. ISSN 00220248. DOI 10.1016/0022-0248(91)90036-5.

[6] H. Hirata and K. Hoshikawa. Three-dimensional numerical analyses of the effects of a cusp magnetic field on the flows, oxygen transport and heat transfer in a Czochralski silicon melt. Journal of Crystal Growth, 125: 181-207, 1992. ISSN 00220248. DOI 10.1016/0022-0248(92)90333-E.

[7] L. Liu, S. Nakano, and K. Kakimoto. Investigation of oxygen distribution in electromagnetic CZSi melts with a transverse magnetic field using 3d global modeling. Journal of Crystal Growth, 299:48-58, 2007. ISSN 00220248. DOI 10.1016/j.jcrysgro.2006.10.247. 
[8] M. Cablea, K. Zaidat, A. Gagnoud, A. Nouri, and Y. Delannoy. Directional solidification of silicon under the influence of travelling magnetic field. Journal of Crystal Growth, 401:883-887, 2014. ISSN 00220248. DOI 10.1016/j.jcrysgro.2013.11.062.

[9] K. Dadzis, D. Vizman, and J. Friedrich. Unsteady coupled 3D calculations of melt flow, interface shape, and species transport for directional solidification of silicon in a traveling magnetic field. Journal of Crystal Growth, 367:77-87, 2013. ISSN 00220248. DOI 10.1016/j.jcrysgro.2012.12.135.

[10] N. Dropka, C. Frank-Rotsch, and P. Rudolph. Numerical study on stirring of large silicon melts by Carousel magnetic fields. Journal of Crystal Growth, 354:1-8, 2012. ISSN 00220248. DOI 10.1016/j.jcrysgro.2012.05.037.

[11] C. Tanasie, D. Vizman, and J. Friedrich. Numerical study of the influence of different types of magnetic fields on the interface shape in directional solidification of multi-crystalline silicon ingots. Journal of Crystal Growth, 318:293-297, 2011. ISSN 00220248. DOI 10.1016/j.jcrysgro.2010.11.034.

[12] S. Dumitrica, D. Vizman, J.P. Garandet, and A. Popescu. Numerical studies on a type of mechanical stirring in directional solidification method of multicrystalline silicon for photovoltaic applications. Journal of Crystal Growth, 360:76-80, 2012. ISSN 00220248. DOI 10.1016/j.jcrysgro.2012.01.011.

[13] J. Aubin, D.F. Fletcher, and C. Xuereb. Modeling turbulent flow in stirred tanks with CFD: the influence of the modeling approach, turbulence model and numerical scheme. Experimental Thermal and Fluid Science, 28:431-445, 2004. ISSN 08941777. DOI 10.1016/j.expthermflusci.2003.04.001.

[14] A. Brucato, M. Ciofalo, F. Grisafi, and G. Micale. Numerical prediction of flow fields in baffled stirred vessels: a comparison of alternative modelling approaches. Chemical Engineering Science, 53:3653-3684, 1998. DOI 10.1016/S0009-2509(98)00149-3.

[15] J. L. Camacho-Martínez, M. A. Ramírez-Argáez, R. Zenit-Camacho, A. Juárez-Hernández, J. D. O. BarceinasSánchez, and G. Trápaga-Martínez. Physical Modelling of an Aluminium Degassing Operation with Rotating Impellers - A Comparative Hydrodynamic Analysis. Materials and Manufacturing Processes, 25:581-591, 2010. ISSN 1042-6914, 1532-2475. DOI 10.1080/10426910903367386.

[16] V.S. Warke, G. Tryggvason, and M.M. Makhlouf. Mathematical modeling and computer simulation of molten metal cleansing by the rotating impeller degasser - Part I. Fluid flow. Journal of Materials Processing Technology, 168:112-118, 2005. ISSN 09240136. DOI 10.1016/j.jmatprotec.2004.10.017.

[17] V.S. Warke, S. Shankar, and M.M. Makhlouf. Mathematical modeling and computer simulation of molten aluminum cleansing by the rotating impeller degasser - Part II. Removal of hydrogen gas and solid particles. Journal of Materials Processing Technology, 168:119-126, 2005. ISSN 09240136. DOI 10.1016/j.jmatprotec.2004.10.016.

[18] C. Audoin, M. Albaric, S. Caron, and M. Chatelain. FR3040644 (A1) - Device For Mechanically Stirring A Molten Metal For A Directed Solidification Method, 2017. http://basesbrevets.inpi.fr/fr/document/FR3040644.html.

[19] W. Dridi, D. Henry, and H. Ben Hadid. Influence of acoustic streaming on the stability of melt flows in horizontal Bridgman configurations. Journal of Crystal Growth, 310:1546-1551, 2008. ISSN 00220248. DOI 10.1016/j.jcrysgro.2007.11.014.

[20] G.N. Kozhemyakin, L.V. Nemets, and A.A. Bulankina. Simulation of ultrasound influence on melt convection for the growth of GaxIn1-xSb and Si single crystals by the Czochralski method. Ultrasonics, 54:2165-2168, 2014. ISSN 0041624X. DOI 10.1016/j.ultras.2014.06.006.

[21] B. Moudjed, V. Botton, D. Henry, H. Ben Hadid, and J.P. Garandet. Scaling and dimensional analysis of acoustic streaming jets. Physics of Fluids, 26:093602, 2014. ISSN 1070-6631, 1089-7666. DOI 10.1063/1.4895518.

[22] R.A. Negrila, A. Popescu, and D. Vizman. Numerical and experimental modeling of melt flow in a directional solidification configuration under the combined influence of electrical current and magnetic field. European Journal of Mechanics - B/Fluids, 52:147-159, 2015. ISSN 09977546. DOI 10.1016/j.euromechflu.2015.03.001. 
[23] J. Y. Luo, R. I. Issa, and A. D. Gosman. Prediction of impeller induced flows in mixing vessels using multiple frames of reference. Institution of Chemical Engineers Symposium Series, Univ Cambridge, England, 1994. Inst. Chemical Engineers. ISBN 0-85295-329-1.

[24] J. B. Joshi, N. K. Nere, C. V. Rane, B. N. Murthy, C. S. Mathpati, A. W. Patwardhan, and V. V. Ranade. CFD simulation of stirred tanks: Comparison of turbulence models (Part II: Axial flow impellers, multiple impellers and multiphase dispersions). The Canadian Journal of Chemical Engineering, 89:754-816, 2011. ISSN 00084034. DOI 10.1002/cjce.20465.

[25] S. Malik, E. Lévêque, M. Bouaifi, L. Gamet, E. Flottes, S. Simoëns, and M. El-Hajem. Shear improved Smagorinsky model for large eddy simulation of flow in a stirred tank with a Rushton disk turbine. Chemical Engineering Research and Design, 108:69-80, 2016. ISSN 02638762. DOI 10.1016/j.cherd.2016.02.035.

[26] P. Mavros. Flow Visualization in Stirred Vessels. Chemical Engineering Research and Design, 79:113-127, 2001. ISSN 02638762. DOI 10.1205/02638760151095926.

[27] J. Aubin, N. Le Sauze, J. Bertrand, D.F. Fletcher, and C. Xuereb. PIV measurements of flow in an aerated tank stirred by a down- and an up-pumping axial flow impeller. Experimental Thermal and Fluid Science, 28: 447-456, 2004. ISSN 08941777. DOI 10.1016/j.expthermflusci.2001.12.001.

[28] S. Bugay, R. Escudie, and A. Line. Experimental analysis of hydrodynamics in axially agitated tank. AIChE journal, 48:463-475, 2002. DOI 10.1002/aic.690480306.

[29] J.F. Hall, M. Barigou, M.J.H. Simmons, and E.H. Stitt. A PIV study of hydrodynamics in gas-liquid high throughput experimentation (HTE) reactors with eccentric impeller configurations. Chemical Engineering Science, 60:6403-6413, 2005. ISSN 00092509. DOI 10.1016/j.ces.2005.03.044.

[30] S. Kaddeche, H.Ben Hadid, and D. Henry. Macrosegregation and convection in the horizontal Bridgman configuration I. Dilute alloys. Journal of Crystal Growth, 135:341-353, 1994. ISSN 00220248. DOI 10.1016/00220248(94)90761-7.

[31] M.P. Bellmann and M. M'Hamdi. Effect of flow pattern on the segregation of impurities in vertical Bridgman growth of multi-crystalline silicon. Journal of Crystal Growth, 362:93-98, 2013. ISSN 00220248. DOI 10.1016/j.jcrysgro.2011.10.055.

[32] Y.Y. Teng, J.C. Chen, C.W. Lu, and C.Y. Chen. The carbon distribution in multicrystalline silicon ingots grown using the directional solidification process. Journal of Crystal Growth, 312:1282-1290, 2010. ISSN 00220248. DOI 10.1016/j.jcrysgro.2009.11.020.

[33] J. Altenberend, Y. Delannoy, A. Nehari, G. Chichignoud, and K. Zaidat. Modelling and experiments for the convecto-diffusive removal of impurities from the solidification front. Journal of Crystal Growth, 458:80-86, 2017. ISSN 00220248. DOI 10.1016/j.jcrysgro.2016.11.052.

[34] J.P. Garandet, N. Kaupp, D. Pelletier, and Y. Delannoy. Solute segregation in a lid driven cavity: Effect of the flow on the boundary layer thickness and solute segregation. Journal of Crystal Growth, 340:149-155, 2012. ISSN 00220248. DOI 10.1016/j.jcrysgro.2011.11.071.

[35] J. P. Garandet, T. Duffar, and J. J. Favier. On the scaling analysis of the solute boundary layer in idealized growth configurations. Journal of crystal growth, 106:437-444, 1990. DOI 10.1016/0022-0248(90)90090-8.

[36] M. Chatelain, S. Rhouzlane, V. Botton, M. Albaric, D. Henry, S. Millet, D. Pelletier, and J.P. Garandet. Towards wall functions for the prediction of solute segregation in plane front directional solidification. Journal of Crystal Growth, 475:55-69, 2017. ISSN 00220248. DOI 10.1016/j.jcrysgro.2017.05.019.

[37] S. Kaddeche, J.P. Garandet, D. Henry, H. Ben Hadid, and A. Mojtabi. On the effect of natural convection on solute segregation in the horizontal Bridgman configuration: Convergence of a theoretical model with numerical and experimental data. Journal of Crystal Growth, 409:89-94, 2014. ISSN 00220248. DOI 10.1016/j.jcrysgro.2014.10.009.

[38] C. J. Chang and R. A. Brown. Radial segregation induced by natural convection and melt/solid interface shape in vertical bridgman growth. Journal of Crystal Growth, 63:343-364, 1983. ISSN 00220248. DOI 10.1016/0022-0248(83)90225-7. 
[39] J. J. Derby and A. Yeckel. Modeling of Crystal Growth Processes. In Crystal Growth - From Fundamentals to Technology, pages 143-167. Elsevier, 2004. ISBN 978-0-444-51386-1. DOI 10.1016/B978-044451386-1/50008-8.

[40] Q. Xiao, S. Kuppurao, A. Yeckel, and J. J. Derby. On the effects of ampoule tilting during vertical Bridgman growth: three-dimensional computations via a massively parallel, finite element method. Journal of Crystal Growth, 167:292-304, 1996. ISSN 00220248. DOI 10.1016/0022-0248(96)00231-X.

[41] Y. Delannoy and K. Zaidat. Modelling the segregation of impurities during solidification with turbulent electromagnetic stirring. In The 7 th International Conference on Electromagnetic Processing of Materials, pages 217-220, 2012.

[42] F.B. Santara. Cristallisation du silicium photovoltä̈que sous induction électromagnétique: étude d'une vanne de rétention et de la ségrégation sous brassage. Ph. D. Thesis, Institut National Polytechnique de Grenoble-INPG, 2010.

[43] L.O. Wilson. On interpreting a quantity in the Burton, Prim and Slichter equation as a diffusion boundary layer thickness. Journal of Crystal Growth, 44:247-250, 1978. ISSN 00220248. DOI 10.1016/0022-0248(78)90199-9.

[44] ANSYS Inc. ANSYS FLUENT 14.0 - Theory Guide, 2011.

[45] D.B. Spalding. Concentration fluctuations in a round turbulent free jet. Chemical Engineering Science, 26: 95-107, 1971. ISSN 00092509. DOI 10.1016/0009-2509(71)86083-9.

[46] Y. Tominaga and T. Stathopoulos. Turbulent Schmidt numbers for CFD analysis with various types of flowfield. Atmospheric Environment, 41:8091-8099, 2007. ISSN 13522310. DOI 10.1016/j.atmosenv.2007.06.054.

[47] M. Chatelain, M. Albaric, D. Pelletier, and V. Botton. Solute segregation in directional solidification: Scaling analysis of the solute boundary layer coupled with transient hydrodynamic simulations. Journal of Crystal Growth, 430:138-147, 2015. ISSN 00220248. DOI 10.1016/j.jcrysgro.2015.08.013.

[48] J.P. Garandet, S. Corre, S. Kaddeche, and T. Alboussière. The influence of convection on the duration of the initial solute transient in alloy crystal growth. Journal of Crystal Growth, 209:970-982, 2000. ISSN 00220248. DOI 10.1016/S0022-0248(99)00630-2.

[49] P. Meunier, T. Leweke, R. Lebescond, B. Van Aughem, and C. Wang. DPIVsoft User Guide, 2004. URL https://www.irphe.fr/ meunier/.

[50] P. Meunier and T. Leweke. Analysis and treatment of errors due to high velocity gradients in particle image velocimetry. Experiments in Fluids, 35:408-421, 2003. ISSN 0723-4864, 1432-1114. DOI 10.1007/s00348-0030673-2.

[51] J.P. Garandet. Microsegregation in crystal growth from the melt: an analytical approach. Journal of Crystal Growth, 131:431-438, 1993. ISSN 00220248. DOI 10.1016/0022-0248(93)90192-Y.

[52] F.Z. Haddad, J.P. Garandet, D Henry, and H.Ben Hadid. Analysis of the unsteady segregation in crystal growth from a melt Part II: Fluctuating convection velocity. Journal of Crystal Growth, 220:166-175, 2000. ISSN 00220248. DOI 10.1016/S0022-0248(00)00587-X. 